\title{
A Note on the Eigenvalues for Periodic Three-Dimensional Jacobi-Perron Algorithms
}

\author{
By \\ Fritz Schweiger \\ (Vorgelegt in der Sitzung der math.-nat. Klasse am 19. Januar 2006 \\ durch das k. M. Fritz Schweiger)
}

\begin{abstract}
In their profound study on the connections between Lyapunov theory and approximation properties of Jacobi-Perron algorithm BROISE-ALAMICHEL and GUIVARC'H 2001 proved a generalization of an inequality due to PALEY and URSELL [2]. In this note this inequality is slightly refined for dimension $n=3$. This shows that for the eigenvalues $\sigma_{0}>\left|\sigma_{1}\right| \geq\left|\sigma_{2}\right| \geq\left|\sigma_{3}\right|$ of a periodic expansion the inequality $\left|\sigma_{1} \sigma_{2}\right|<1$ is true. Furthermore it allows a more direct proof for the inequality $\lambda_{1}+\lambda_{2}<0$ where $\lambda_{0}>\lambda_{1}>\lambda_{2}>\lambda_{3}$ are the Lyapunov exponents of the algorithm.
\end{abstract}

Mathematics Subject Classification (2000): 11K55, 11J13, 11J70.

Key words: Multidimensional continued fractions, periodic expansions, Lyapunov exponents.

\section{Introduction}

Let

$$
\begin{gathered}
T\left(x_{1}, x_{2}, x_{3}\right)=\left(\frac{x_{2}}{x_{1}}-a, \frac{x_{3}}{x_{1}}-b, \frac{1}{x_{1}}-c\right), \quad a(x)=\left[\frac{x_{2}}{x_{1}}\right], \\
b(x)=\left[\frac{x_{3}}{x_{1}}\right], \quad c(x)=\left[\frac{1}{x_{1}}\right]
\end{gathered}
$$


denote the three-dimensional map related to Jacobi-Perron algorithm (PERron [3], SchweIGer [4, 6]). Define

$$
\left(a_{s}, b_{s}, c_{s}\right)=\left(a\left(T^{s-1} x\right), b\left(T^{s-1} x\right), c\left(T^{s-1} x\right)\right), \quad 1 \leq s .
$$

Note that the digits satisfy the so-called Perron conditions

$$
0 \leq a_{s} \leq c_{s}, \quad 0 \leq b_{s} \leq c_{s}, \quad 1 \leq c_{s} .
$$

Furthermore: If $a_{s}=c_{s}$ then $1 \leq b_{s+1}$. If $b_{s}=c_{s}$ then $a_{s+1} \leq b_{s+1}$. If $b_{s}=c_{s}$ and $a_{s+1}=b_{s+1}$ then $1 \leq a_{s+2}$.

We introduce the matrices

$$
\beta^{(s)}:=\left(\begin{array}{cccc}
c_{s} & 0 & 0 & 1 \\
1 & 0 & 0 & 0 \\
a_{s} & 1 & 0 & 0 \\
b_{s} & 0 & 1 & 0
\end{array}\right)
$$

and

$$
\beta^{(1)} \cdots \beta^{(s)}:=\left(\begin{array}{llll}
A_{0}^{(s+4)} & A_{0}^{(s+1)} & A_{0}^{(s+2)} & A_{0}^{(s+3)} \\
A_{1}^{(s+4)} & A_{1}^{(s+1)} & A_{1}^{(s+2)} & A_{1}^{(s+3)} \\
A_{2}^{(s+4)} & A_{2}^{(s+1)} & A_{2}^{(s+2)} & A_{2}^{(s+3)} \\
A_{3}^{(s+4)} & A_{3}^{(s+1)} & A_{3}^{(s+2)} & A_{3}^{(s+3)}
\end{array}\right) .
$$

From this notation we read the recursion relation

$$
A_{\alpha}^{(s+4)}=c_{s} A_{\alpha}^{(s+3)}+b_{s} A_{\alpha}^{(s+2)}+a_{s} A_{\alpha}^{(s+1)}+A_{\alpha}^{(s)}, \quad \alpha \in\{0,1,2,3\} .
$$

The following expansion is worth to be stated as a separate lemma.

Lemma (BROISE-ALAMICHEL and GUIVARC'H [1]).

$$
\begin{aligned}
A_{0}^{(s+4)}= & \left(c_{s}-1\right) A_{0}^{(s+3)}+\left(c_{s-1}+b_{s}-1\right) A_{0}^{(s+2)} \\
& +\left(c_{s-2}+b_{s-1}+a_{s}-1\right) A_{0}^{(s+1)}+\left(c_{s-3}+b_{s-2}+a_{s-1}+1\right) A_{0}^{(s)} \\
& +\left(b_{s-3}+a_{s-2}+1\right) A_{0}^{(s-1)}+\left(a_{s-3}+1\right) A_{0}^{(s-2)}+A_{0}^{(s-3)} .
\end{aligned}
$$

We denote by

$$
[s+i, s+j, s+k]_{\alpha, \beta}=\operatorname{det}\left(\begin{array}{lll}
A_{0}^{(s+i)} & A_{0}^{(s+j)} & A_{0}^{(s+k)} \\
A_{\alpha}^{(s+i)} & A_{\alpha}^{(s+j)} & A_{\alpha}^{(s+k)} \\
A_{\beta}^{(s+i)} & A_{\beta}^{(s+j)} & A_{\beta}^{(s+k)}
\end{array}\right)
$$

the relevant determinants. Since the choice of $\alpha, \beta \in\{1,2,3\}$ is not important we drop these indices. 
The following recursion relations are valid,

$$
\begin{aligned}
& {[s+4, s+1, s+2]=c_{s}[s+3, s+1, s+2]+[s, s+1, s+2],} \\
& {[s+4, s+1, s+3]=b_{s}[s+2, s+1, s+3]+[s, s+1, s+3],} \\
& {[s+4, s+2, s+3]=a_{s}[s+1, s+2, s+3]+[s, s+2, s+3] .}
\end{aligned}
$$

These relations lead to the following useful expansion,

$$
\begin{aligned}
{[s+4, s+2, s+3]=} & a_{s}[s+1, s+2, s+3]+b_{s-1}[s+1, s, s+2] \\
& +c_{s-2}[s+1, s-1, s]+[s-2, s-1, s] .
\end{aligned}
$$

We introduce the quantity

$$
\rho_{s}:=\frac{|[s, s-1, s-2]|}{A_{0}^{(s)}}, \quad s \geq 4 .
$$

\section{Theorem.}

$$
|[s+4, s+2, s+3]| \leq A_{0}^{(s+4)}\left(1-\frac{A_{0}^{(s-2)}}{A_{0}^{(s+4)}}\right) \max _{s-2 \leq t \leq s+3} \rho_{t} .
$$

Proof. The proof will be given by induction and by considering several cases. The relation

$$
\begin{aligned}
{[s+4, s+2, s+3]=} & a_{s}[s+1, s+2, s+3]+b_{s-1}[s+1, s, s+2] \\
& +c_{s-2}[s+1, s-1, s]+[s-2, s-1, s]
\end{aligned}
$$

immediately gives

$$
\begin{aligned}
|[s+4, s+2, s+3]| \leq & \left(a_{s} A_{0}^{(s+3)}+b_{s-1} A_{0}^{(s+2)}\right. \\
& \left.+c_{s-2} A_{0}^{(s+1)}+A_{0}^{(s)}\right) \max _{s \leq t \leq s+3} \rho_{t} .
\end{aligned}
$$

(1) If the following three conditions are satisfied, namely $a_{s} \leq c_{s}-1, \quad b_{s-1} \leq c_{s-1}+b_{s}-1, \quad c_{s-2} \leq c_{s-2}+b_{s-1}+a_{s}-1$, then a comparison with

$$
\begin{aligned}
A_{0}^{(s+4)}= & \left(c_{s}-1\right) A_{0}^{(s+3)}+\left(c_{s-1}+b_{s}-1\right) A_{0}^{(s+2)} \\
& +\left(c_{s-2}+b_{s-1}+a_{s}-1\right) A_{0}^{(s+1)}+\left(c_{s-3}+b_{s-2}+a_{s-1}+1\right) A_{0}^{(s)} \\
& +\left(b_{s-3}+a_{s-2}+1\right) A_{0}^{(s-1)}+\left(a_{s-3}+1\right) A_{0}^{(s-2)}+A_{0}^{(s-3)}
\end{aligned}
$$

shows that

$$
|[s+4, s+2, s+3]| \leq A_{0}^{(s+4)}\left(1-\frac{A_{0}^{(s)}}{A_{0}^{(s+4)}}\right) \max _{s \leq t \leq s+3} \rho_{t} .
$$


(2) Now assume that $c_{s}-1<a_{s}$. Then by Perron conditions we have $a_{s}=c_{s}$. Then we calculate

$$
\begin{aligned}
{[s+4, s+2, s+3]=} & c_{s}[s+1, s+2, s+3]+[s, s+2, s+3] \\
= & \left(c_{s}-1\right)[s+1, s+2, s+3] \\
& +[s+1, s+2, s+3]+[s, s+2, s+3] \\
= & \left(c_{s}-1\right)[s+1, s+2, s+3] \\
& +\left(a_{s-1}-b_{s-1}\right)[s, s+1, s+2] \\
& +\left(b_{s-2}-c_{s-2}\right)[s, s-1, s+1] \\
& +\left(1+c_{s-3}\right)[s, s-2, s-1]+[s-3, s-2, s-1] .
\end{aligned}
$$

This gives the estimate

$$
\begin{aligned}
|[s+4, s+2, s+3]| \leq & \max _{s-1 \leq t \leq s+3} \rho_{t}\left(\left(c_{s}-1\right) A_{0}^{(s+3)}+\left|a_{s-1}-b_{s-1}\right| A_{0}^{(s+2)}\right. \\
& \left.+\left|b_{s-2}-c_{s-2}\right| A_{0}^{(s+1)}+\left(1+c_{s-3}\right) A_{0}^{(s)}+A_{0}^{(s-1)}\right) .
\end{aligned}
$$

We will show that the inequalities

$\left|a_{s-1}-b_{s-1}\right| \leq c_{s-1}+b_{s}-1, \quad\left|b_{s-2}-c_{s-2}\right| \leq c_{s-2}+b_{s-1}+a_{s}-1$

are satisfied. Then a comparison with the expansion in the lemma shows

$$
|[s+4, s+2, s+3]| \leq A_{0}^{(s+4)}\left(1-\frac{A_{0}^{(s-2)}}{A_{0}^{(s+4)}}\right) \max _{s-2 \leq t \leq s+3} \rho_{t} .
$$

Suppose that $a_{s-1}-b_{s-1}>c_{s-1}+b_{s}-1$. Then $c_{s-1}+1 \geq a_{s-1}+1$ $>c_{s-1}+b_{s-1}+b_{s}$. Then $b_{s-1}=b_{s}=0$ and $c_{s-1}=a_{s-1}$. But the last condition implies $1 \leq b_{s}$, a contradiction.

If $b_{s-1}-a_{s-1}>c_{s-1}+b_{s}-1$ then $c_{s-1}+1 \geq b_{s-1}+1>c_{s-1}+$ $a_{s-1}+b_{s}$. Hence $a_{s-1}=b_{s}=0$ and $b_{s-1}=c_{s-1}$. But $b_{s-1}=c_{s-1}$ implies $a_{s} \leq b_{s}$, hence $a_{s}=0$, a contradiction.

If $c_{s-2}-b_{s-2}>c_{s-2}+b_{s-1}+a_{s}-1$ then $1>b_{s-2}+b_{s-1}+a_{s}$. This leads to $b_{s-2}=b_{s-1}=a_{s}=0$ which again contradicts $a_{s}=c_{s}$.

(3) Therefore from now on we assume $a_{s} \leq c_{s}-1$. However, there are two conditions left which could be violated.

(3.1) We first assume $c_{s} \geq 2$.

(3.1.1) We consider the case

$$
b_{s-1}>b_{s}+c_{s-1}-1 .
$$


From $b_{s-1} \leq c_{s-1}$ we get $b_{s-1}=c_{s-1}$ and $b_{s}=0$ and therefore also $a_{s}=0$. Then we have the recursion

$$
\begin{aligned}
{[s+4, s+2, s+3]=} & b_{s-1}[s+1, s, s+2]+c_{s-2}[s+1, s-1, s] \\
& +[s-2, s-1, s] .
\end{aligned}
$$

Then we estimate

$$
|[s+4, s+2, s+3]| \leq\left(c_{s-1} A_{0}^{(s+2)}+c_{s-2} A_{0}^{(s+1)}+A_{0}^{(s)}\right)\left(\max _{s \leq t \leq s+2} \rho_{t}\right) .
$$

This expression must be compared with

$$
\begin{aligned}
A_{0}^{(s+4)} \geq & \left(c_{s}-1\right) A_{0}^{(s+3)}+\left(c_{s-1}-1\right) A_{0}^{(s+2)} \\
& +\left(c_{s-2}+c_{s-1}-1\right) A_{0}^{(s+1)}+\left(c_{s-3}+1\right) A_{0}^{(s)} .
\end{aligned}
$$

Since

$$
c_{s-1} A_{0}^{(s+2)} \leq A_{0}^{(s+3)}+\left(c_{s-1}-1\right) A_{0}^{(s+2)}
$$

we obtain

$$
|[s+4, s+2, s+3]| \leq A_{0}^{(s+4)}\left(1-\frac{A_{0}^{(s)}}{A_{0}^{(s+4)}}\right) \max _{s \leq t \leq s+3} \rho_{t} .
$$

(3.1.2) Next suppose that

$$
c_{s-2}>c_{s-2}+b_{s-1}+a_{s}-1 .
$$

Then $a_{s}=b_{s-1}=0$ and we find

$$
[s+4, s+2, s+3]=c_{s-2}[s+1, s-1, s]+[s-2, s-1, s] .
$$

This leads to a comparison of

$$
c_{s-2} A_{0}^{(s+1)}+A_{0}^{(s)}
$$

with

$A_{0}^{(s+4)} \geq\left(c_{s}-1\right) A_{0}^{(s+3)}+\left(c_{s-1}+b_{s}-1\right) A_{0}^{(s+2)}+\left(c_{s-2}-1\right) A_{0}^{(s+1)}+A_{0}^{(s)}$.

Since

$$
c_{s-2} A_{0}^{(s+1)} \leq A_{0}^{(s+3)}+\left(c_{s-2}-1\right) A_{0}^{(s+1)}
$$

we get the same estimate as before.

(3.2) The remaining case is $c_{s}=1$.

(3.2.1)

$$
b_{s-1}>b_{s}+c_{s-1}-1
$$


leads to $b_{s-1}=c_{s-1}$ and $b_{s}=0$ and $a_{s}=0$. We expand the relation

$$
\begin{aligned}
{[s+4, s+2, s+3]=} & b_{s-1}[s+1, s, s+2]+c_{s-2}[s+1, s-1, s] \\
& +[s-2, s-1, s]
\end{aligned}
$$

to obtain

$$
\begin{aligned}
{[s+4, s+2, s+3]=} & \left(b_{s-1}-1\right)[s+1, s, s+2] \\
& +\left(c_{s-2}-a_{s-2}\right)[s+1, s-1, s] \\
& -b_{s-3}[s-1, s-2, s]-c_{s-4}[s-1, s-3, s-2] \\
& +[s-2, s-1, s]-[s-4, s-3, s-2] .
\end{aligned}
$$

As before we compare this relation with the expansion

$$
\begin{aligned}
A_{0}^{(s+4)}= & \left(c_{s}-1\right) A_{0}^{(s+3)}+\left(c_{s-1}+b_{s}-1\right) A_{0}^{(s+2)} \\
& +\left(c_{s-2}+c_{s-1}+a_{s}-1\right) A_{0}^{(s+1)}+\left(c_{s-3}+b_{s-2}+a_{s-1}+1\right) A_{0}^{(s)} \\
& +\left(b_{s-3}+a_{s-2}+1\right) A_{0}^{(s-1)}+\left(a_{s-3}+1\right) A_{0}^{(s-2)}+A_{0}^{(s-3)} .
\end{aligned}
$$

Obviously, $c_{s-2}-a_{s-2} \leq c_{s-2}+c_{s-1}+a_{s}-1$ is true. The remaining critical estimate is

$$
\begin{aligned}
\left(b_{s-3}+1\right) A_{0}^{(s)}+c_{s-4} A_{0}^{(s-1)} \leq & \left(c_{s-3}+b_{s-2}+a_{s-1}+1\right) A_{0}^{(s)} \\
& +\left(b_{s-3}+a_{s-2}+1\right) A_{0}^{(s-1)}
\end{aligned}
$$

or equivalently

$b_{s-3} A_{0}^{(s)}+c_{s-4} A_{0}^{(s-1)} \leq\left(c_{s-3}+b_{s-2}+a_{s-1}\right) A_{0}^{(s)}+\left(b_{s-3}+a_{s-2}\right) A_{0}^{(s-1)}$.

If $b_{s-3}=c_{s-3}$ then we obtain $a_{s-2} \leq b_{s-2}$. If $b_{s-2}=0$ then $a_{s-2}=0$ and hence $a_{s-1} \geq 1$. The other case is $b_{s-1} \geq 1$. In both cases we obtain

$$
c_{s-4} A_{0}^{(s-1)} \leq A_{0}^{(s)}+A_{0}^{(s-1)} .
$$

Hence

$$
|[s+4, s+2, s+3]| \leq A_{0}^{(s+4)}\left(1-\frac{A_{0}^{(s-1)}}{A_{0}^{(s+4)}}\right) \max _{s-1 \leq t \leq s+3} \rho_{t} .
$$

If $b_{s-3}<c_{s-3}$ then we get again

$$
c_{s-4} A_{0}^{(s-1)} \leq A_{0}^{(s)}+A_{0}^{(s-1)}
$$

and the same result. 
(3.2.2) If

$$
c_{s-2}>c_{s-2}+b_{s-1}+a_{s}-1
$$

then $b_{s-1}=a_{s}=0$. Note that in this case clearly $b_{s-1} \leq b_{s}+c_{s-1}-1$. We look at

$$
[s+4, s+2, s+3]=c_{s-2}[s+1, s-1, s]+[s-2, s-1, s]
$$

and compare this with

$$
\begin{aligned}
A_{0}^{(s+4)} \geq & \left(c_{s-2}-1\right) A_{0}^{(s+1)}+\left(c_{s-3}+1\right) A_{0}^{(s)}+\left(b_{s-3}+1\right) A_{0}^{(s-1)} \\
& +\left(a_{s-3}+1\right) A_{0}^{(s-2)}+A_{0}^{(s-3)}
\end{aligned}
$$

which is equivalent to

$$
A_{0}^{(s+4)} \geq c_{s-2} A_{0}^{(s+1)}+A_{0}^{(s)}+A_{0}^{(s-1)}+A_{0}^{(s-2)} .
$$

This leads to the estimate

$$
|[s+4, s+2, s+3]| \leq A_{0}^{(s+4)}\left(1-\frac{A_{0}^{(s-1)}}{A_{0}^{(s+4)}}\right) \max _{s-2 \leq t \leq s+1} \rho_{t} .
$$

For a periodic algorithm with eigenvalues $\sigma_{0}, \sigma_{1}, \sigma_{2}, \sigma_{3}$ ordered in a way such that $\sigma_{0}>\left|\sigma_{1}\right| \geq\left|\sigma_{2}\right| \geq\left|\sigma_{3}\right|$ the following corollary follows.

Corollary 1. $\left|\sigma_{1} \sigma_{2}\right|<1$.

Proof. Note that for a periodic algorithm with period length $p$ the relations

$$
A_{0}^{(s p+4)}+A_{1}^{(s p+1)}+A_{2}^{(s p+2)}+A_{3}^{(s p+3)} \sim \sigma_{0}^{s}
$$

and

$$
\begin{aligned}
& {[s p+4, s p+1, s p+2]_{0,1,2}+[s p+1, s p+2, s p+3]_{1,2,3}} \\
& \quad+[s p+4, s p+2, s p+3]_{0,2,3}+[s p+4, s p+1, s p+3]_{0,1,3} \\
& \quad \sim\left|\sigma_{0} \sigma_{1} \sigma_{2}\right|^{s} .
\end{aligned}
$$

Here the quantities $[s+i, s+j, s+k]_{\alpha, \beta, \gamma}$ are defined as

$$
[s+i, s+j, s+k]_{\alpha, \beta, \gamma}=\operatorname{det}\left(\begin{array}{ccc}
A_{\alpha}^{(s+i)} & A_{\alpha}^{(s+j)} & A_{\alpha}^{(s+k)} \\
A_{\beta}^{(s+i)} & A_{\beta}^{(s+j)} & A_{\beta}^{(s+k)} \\
A_{\gamma}^{(s+i)} & A_{\gamma}^{(s+j)} & A_{\gamma}^{(s+k)}
\end{array}\right) .
$$


Clearly the theorem of the paper extends to these numbers. Since the algorithm is periodic there is a constant $q<1$ such that

$$
1-\frac{A_{0}^{(s-2)}}{A_{0}^{(s+4)}} \leq q<1 .
$$

If $\lambda_{0}, \lambda_{1}, \lambda_{2}, \lambda_{3}$ are the four Lyapunov exponents the following result can be proved by applying the ergodic theorem.

Corollary 2. $\lambda_{0}+\lambda_{3}>0$.

Proof. The proof closely follows SCHWEIGER [5] (see also SCHWEIGER [4]). We introduce the quantity

$$
\tau_{s}:=\max _{0 \leq j \leq 5} \rho_{s+j}
$$

Then we find

$$
\tau_{s+6} \leq \max _{0 \leq j \leq 5}\left(1-\frac{A_{0}^{(s+j-2)}}{A_{0}^{(s+j+4)}}\right) \tau_{s} .
$$

Using estimates like

$$
1-\frac{A_{0}^{(s-2)}}{A_{0}^{(s+4)}} \leq 1-\frac{1}{4^{6} c_{s} c_{s-1} c_{s-2} c_{s-3} c_{s-4} c_{s-5}},
$$

we see that the product

$$
\prod_{s=1}^{N}\left(1-\max _{0 \leq j \leq 5}\left(1-\frac{A_{0}^{(s+j-2)}}{A_{0}^{(s+j+4)}}\right)\right)^{1 / N}
$$

can be estimated almost everywhere by using the ergodic theorem. More precisely, there is a constant $\kappa<1$ such that

$$
\prod_{s=1}^{N}\left(1-\max _{0 \leq j \leq 5}\left(1-\frac{A_{0}^{(s+j-2)}}{A_{0}^{(s+j+4)}}\right)\right)^{1 / N} \leq \kappa
$$

holds almost everywhere. Since almost everywhere

$$
\lim _{s \rightarrow \infty} \frac{\log A_{0}^{(s)}}{s}=\lambda_{0}
$$

the proof can be easily completed.

\section{Acknowledgement}

The author likes to thank B. SCHRATZBERGER who is engaged in the project P16964 of the Austrian Science Foundation (Multidimensional Continued Fractions) for helpful comments. 


\section{References}

[1] Broise-Alamichel, A., Guivarc'H, Y. (2001) Exposants caractéristiques de l'algorithme de Jacobi-Perron et de la transformation associée. Ann. Inst. Fourier (Grenoble) 51 (no. 3): 565-686

[2] Paley, R. E. A. C., Ursell, H. D. (1930) Continued fractions in several dimensions. Proc. Cambridge Phil. Soc. 26: 127-144

[3] Perron, O. (1907) Grundlagen für eine Theorie des Jacobischen Kettenbruchalgorithmus. Math. Ann. 64: 1-76

[4] SchweIger, F. (2000) Multidimensional Continued Fractions. Oxford: Oxford University Press

[5] SCHWEIGER, F. (1996) The exponent of convergence for the 2-dimensional JacobiPerron algorithm. In: NowAK, W. G., SchOIsSEnGEIER, J. (eds.) Proceedings of the Conference on Analytic and Elementary Number Theory, pp. 207-213. Institut für Mathematik, Universität für Bodenkultur, Wien

[6] SchweIger, F. (2002) Diophantine properties of multidimensional continued fractions. In: DUBICKAS, A., et al. (eds.) Analytic and Probabilistic Methods in Number Theory. Proc. 3rd International Conference in Honour of J. Kubilius, pp. 242-255. TEV Ltd., Vilnius

Author's address: Fritz Schweiger, Department of Mathematics, University of Salzburg, Hellbrunner Strasse 34, 5020 Salzburg, Austria. E-Mail: fritz.schweiger@ sbg.ac.at. 